ÁMBITOS. № 17 - Año 2008 (pp 59-72)

\title{
LA DIGITALIZACIÓN EN CAMPO TELEVISIVO EUROPEO
}

\author{
Elias Said Hung \\ (Universidad del Norte, Barranquilla-Colombia) \\ saide@uninorte.edu.co
}

Resumen:

El actual reto que surge con el paso progresivo a lo digital y las condiciones particulares en Europa hacen que no se potencie aún más las fortalezas y cualidades existentes en ella. La creciente disposición de cada Estado en asumir un rol pasivo en este complejo puzzle audiovisual, ha generado que la sensación de vacío sea una constante y que se asuma la transición completa de lo analógico a lo digital como algo definitivo, pero nada claro de cómo terminará, cumplido el plazo propuesto para ello en cada país.

Palabras claves: Digitalización, televisión, Europa, SIC, TIC.

\begin{abstract}
:
The current challenge that arises with the progressive step to the digital thing and the particular conditions in Europe make it not to be stronger the strengths and existent qualities in this region. The growing disposition of each States in assuming a passive role in this complex audiovisual puzzle, it has generated that the hole sensation is a constant and that the complete transition is assumed from the analogical scenario to the digital one as something definitive, but anything clear of how will it finish, and the end of the term proposed for it in each country.
\end{abstract}

Key words: Digitalization, television, Europe, Information and Knowledge Society, Information and Knowledge Technology.

\section{INTRODUCCIÓN}

as comunicaciones, a medida que discurrimos en el actual proceso de transición hacia el cruce de caminos que nos termina generando el avance tecnológico en el marco de lo que se conoce como Sociedad de la Información y Conocimiento (SIC), ya no deben ser consideradas como procesos enmarcado en el ámbito cultural, sino también como elementos que contribuyen al desarrollo económico y de la propia organización social en todos sus aspectos (educativo, ocio y transporte, entre otros).

Ante el redimensionamiento del modelo de las comunicaciones tradicionales, las industrias relacionadas con las tecnologías de información y conocimiento (TIC) y el desarrollo de contenido se han convertido en dos de los actores más importantes y 
con mayor crecimiento en la economía mundial actual, hasta hacer que se vaya delineando un nuevo entorno comunicativo caracterizado por contar con la capacidad de generación de un mayor poderío de aquellas actividades relacionadas con el consumo, tratamiento y almacenamiento o distribución de la información (Ramonet, 2000).

El proceso de reajuste que ha traído consigo este nuevo auge de las TIC y el proceso de avance de lo digital sobre lo analógico está trayendo consigo un aumento en la velocidad de desarrollo de las infraestructuras comunicativas que actualmente tenemos ante nosotros, haciendo que se deban ajustar, por ejemplo, leyes como la de la evolución tecnológica de Moore ${ }^{1}$ (Intel, 2007) y la ley de banda ancha en Internet de Charles Guilder ${ }^{2}$, empleado por Fogel (2004), ante los siguientes factores:

1. El acelerado proceso evolutivo de los recursos TIC's en la sociedad actual, las cuales traen consigo la reducción de los tiempos requeridos para la aparición de nuevos componentes informáticos para la generación de soportes y servicios telemáticos que comienzan a abrirse paso ante nosotros.

2. Las desfavorables condiciones de apropiación tecnológica en el mundo y el consiguiente aumento de la brecha digital en las sociedades (Santoyo y Martínez, 2003), impidiéndose así el carácter global en estas leyes.

3. La lenta evolución de contenidos que nutran las potencialidades implícitas en torno a las capacidades técnicas de los avances tecnológicos y el abaratamiento de los servicios actualmente existentes, por falta de asentamiento de un escenario comercial y de contenido estable que facilite la reducción de costes requeridos por los ciudadanos para el contacto con el nuevo horizonte digital que comienza a despuntar.

Lo antes expuesto, hace que nos ubiquemos en un escenario cargado de dudas, ilusiones, miedos y expectativas alrededor de las capacidades que comienzan a percibirse ante las TIC's y la digitalización del sector televisivo.

Las situaciones en que estamos inmersos variarán de acuerdo con los distintos niveles de profundidad permitidos por las condiciones existentes en las diferentes regiones del mundo, las cuales pueden ser palpables a partir de los indicadores de la SIC, medidos por distintos organismos de ámbitos nacionales, regionales y globales, como por ejemplo el Instituto Nacional de Estadística (2006); la Internacional Telecommunication Union (2007, 2007a); la Fundación Telefónica (2005, 2007); la Comisión Europea (2006); la Asociación Española de Empresa de Tecnología de Información (2006); la Comisión del Mercado de las Telecomunicaciones (2007); entre otras. Por ello, es común, aún, encontrarnos debatiendo en torno a la SIC, mientras zonas del planeta conviven con modelos sociales anteriores (agrarios, industriales, post-industriales), sin que esto implique la no imposición progresiva del PTE característico de Era Digital.

\footnotetext{
${ }^{1}$ Esta ley dice que cada 18 meses la potencia del chip en el mundo tiene que duplicarse por dos pero que el precio debe dividirse entre dos.

2 Expone que cada dos años la anchura global de la banda ancha en Internet tiene que ser duplicada.
} 


\section{REFERENTES DEL MUNDO ACTUAL}

Comprender los distintos elementos que dan cuenta del paso de la Sociedad Industrial a la Sociedad de la Información y Conocimiento, amerita el abordaje del proceso denominado como globalización. Pese a que reconocidos investigadores, como por ejemplo, Brünner (1998), Castells, (2005), Beck, (1998), Negroponte (2000), Giddens (2002), por citar algunos la han abordado; ello no impide entenderla como una serie compleja de micro [local] y macro [global] procesos, los cuales entran en acuerdo y en conflicto entre sí de forma constante.

Este confluir y constante proceso de encuentro y des-encuentro entre lo local y lo global, como resultado de la ruptura generada a partir de la disolución de fronteras ante el aumento de flujos informativos, transmitidos de forma transversal en la sociedad; y el creciente fortalecimiento de lo que definimos como Plutarquía Mediática (Said, 2005), formada por un cada vez menor número de grupos empresariales con capacidad de control de una amplia diversidad de sectores relacionados al campo de las telecomunicaciones e informática; entre otros factores, traen consigo un entorno donde lo de ahí afuera, externo al individuo, juega con lo ahí adentro que forma parte de nuestras vidas, donde, empleando un término usado por Sacristán (2003), la actitud de integración cooperativa de grupos sociales entra cada vez más en contacto, lo cual traerá consigo un espacio marcado por una relación asimétrica o no de normas culturales diferentes, donde la no pasividad de los individuos frente a la SIC y conjunto de procesos ejecutados comienzan a constituirse como una opción real, ante los recursos potenciales que brindan las TIC en ellas; haciendo que las culturas adquieran dimensiones caracterizadas por las confluencias y choques de procesos globales y locales, y que las comunidades ganen en influjo sobre la constitución de espacios sociales globales, dentro de sus contextos locales o nacionales.

Por ello, la globalización no sólo debe ser entendida en términos de flujo de información y establecimiento de normas globales de acción de la economía, a través de actores económicos anónimos, sino que debe ser concebida glocalmente, ya que en ella entran en juego procesos como la hibridación cultural (García Canclini, 2001) y la constitución de megalópolis (Olalquiaga, 1991), así como la simultaneidad virtual de acontecimientos no simultáneos, entre otros. Con ello, se cumple lo expuesto claramente por Beck (1998) de que con la globalización corre pareja cada vez más la localización. Este hecho hace que convivamos en una sociedad que nos hace sentir como si estuviésemos en una cinta de ejercicios cuyo botón de apagado está averiado y que progresivamente hace que vayamos más rápido hasta niveles nunca antes experimentados, llevando nuestros límites de percepción y resistencia al máximo.

\section{LA DIGITALIZACIÓN EN EL CAMPO TELEVISIVO EUROPEO}

Bajo los distintos soportes que dan cuenta de la TV digital (satélite, cable, terrestre e Internet), el escenario europeo se está desarrollando alrededor del estándar creado por el consorcio Digital Video Broadcasting (DVB, http://dvb.org/), aplicado para distintas modalidades tecnológicas digitales: DVB-S y DVB-S2 (satélite), DVBC (cable) y DVB-T (TDT). 
Como sucede en el resto del mundo, la era de la digitalización en las aplicaciones de vídeo dirigida al gran público, no ha hecho más que empezar. Si bien el consorcio DVB ha contribuido a la generación de un entorno federalista en Europa para este tema, aún no termina de imponerse un sistema de control de acceso único. Como consecuencia de esto, la lucha entre plataformas digitales constituidas por los diferentes actores económicos en la región han marcado la característica principal alrededor de esta temática, ya que cada uno de ellos tratan de imponer su tecnología en el campo del control de acceso y el tipo de interfaz empleado por los usuarios.

En Europa, a finales de 1970, se comienza a presenciar una crisis generalizada en el modelo empresarial de televisión pública, base fundamental desde la que se desarrolla este sector en la región. Alguna de las principales causas que contribuyeron a este escenario, fueron la creciente erosión de la base financiera tradicionalmente asumida por instituciones radioeléctricas públicas; la expansión del mercado audiovisual y el surgir de nuevos avances y soportes tecnológicos (cable, vídeo, satélite); la pérdida progresiva de terreno del concepto de servicio público en torno al sector audiovisual; y la pérdida de poder de los actores políticos, los cuales han ido asumiendo más la posición de promotores o facilitadores de las condiciones sociales propicias para el desarrollo comercial y el aumento de la influencia de los actores económicos en una mayor cantidad de ámbitos sociales.

Estas causas traen consigo un escenario en el que el debilitamiento generalizado del sector público, la desregulación normativa del sector audiovisual, y la internacionalización de los nuevos operadores privados que comienzan a aparecer en el ámbito regional, se afianzan en el horizonte audiovisual europeo. Es en esta época cuando se llega a un punto de no retorno de este campo, y se da inicio al actual camino liberalizador y de expansión continua, presente a día de hoy.

Con el comienzo de la década de 1990, los procesos iniciados dos décadas atrás en Europa entran a una nueva fase evolutiva, es la etapa de la digitalización. El modelo que comienza a delinearse de forma más clara en esta nueva fase ya no estaba fundamentada en torno a la estrategia de pull publicitaria, sino en nuevos canales de contacto publicitarios, y en la creación de demanda de una nueva generación de contenidos y servicios que comienzan a aparecer en este momento histórico y una mayor valorización de éstos por parte de los usuarios finales. Existe un conjunto de factores que entran en juego para el paso de lo digital en el escenario europeo y sobre todo, para el siguiente cambio del modelo empresarial asumido desde Europa en torno al sector audiovisual: el acelerado y continuo crecimiento de los costes de producción, ante la recurrente necesidad de conquista de un mayor número de audiencia en un mercado cada vez más competitivo; el aumento de los derechos de transmisión, en especial los relativos al ámbito del cine y deportes masivos; la continua y mayor fragmentación del usuario, ante el aumento de canales y las dimensiones de los diferentes mercados nacionales en la región, los cuales imposibilitan el completo sufragio de los costes requeridos solamente con la publicidad; el aumento de una nueva ola de normativas nacionales e internacionales que ejercerán presión dentro del sector comercial audiovisual, con su consiguiente impacto en la reducción de las fuentes de financiamientos. 
Esta nueva etapa termina por abonar el camino a la adopción del modelo de pago directo por el usuario conocido, ya asentado en el contexto europeo. Además, la nueva ola normativa en la Unión Europea, comenzada a partir de 1996, hace que los Estados lleguen a un punto en el que se reconoce una situación de hecho generada por la propia dinámica descrita con anterioridad y sobre todo, se asume la cesión del control público en el cable y satélite, ante el predominio de lo privado en ambos casos, y se comienza a vislumbrar la TDT como el principal soporte digital en el que se preservará el perdido protagonismo de estos actores.

Figura 1. Evolución de los modelos televisivos en Europa.

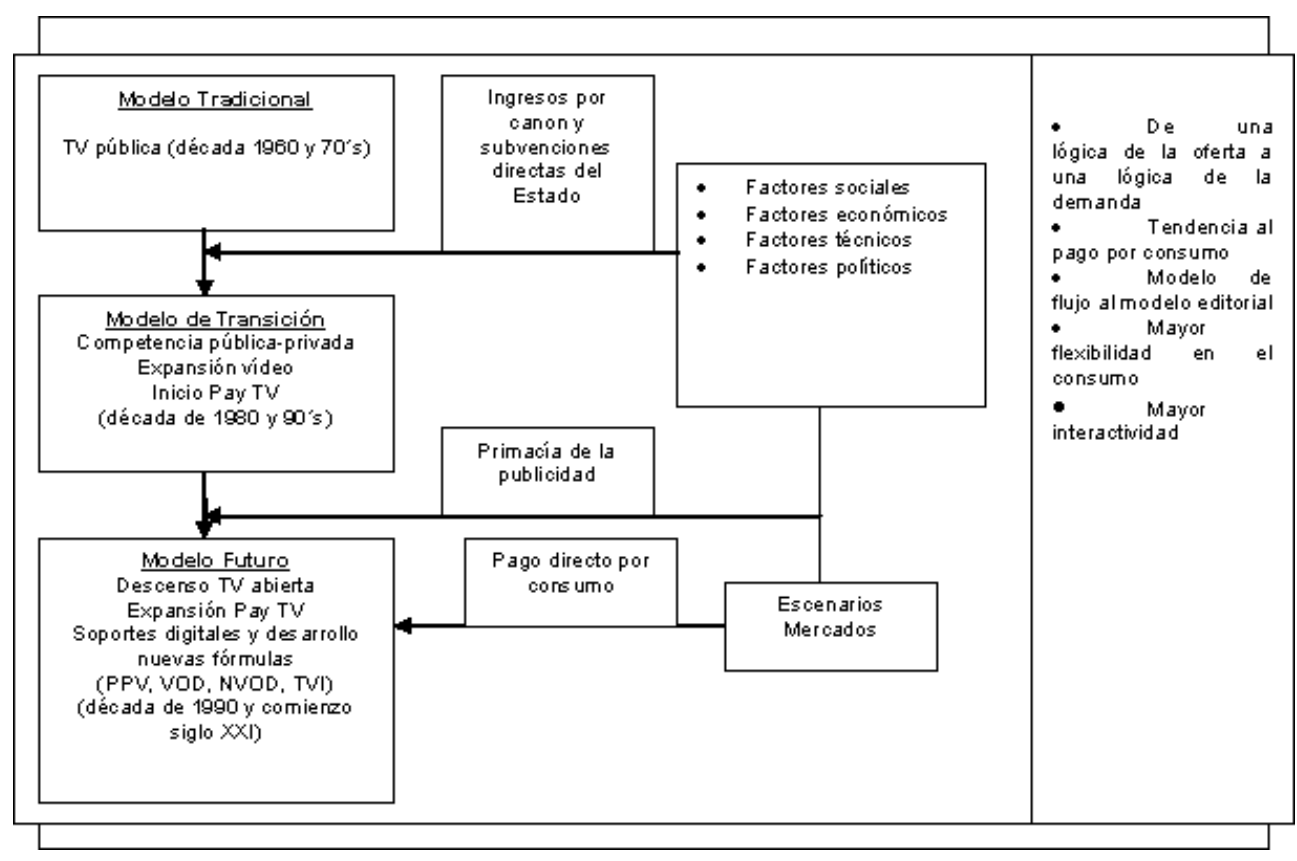

Fuente: ÁLVAREZ, J. Las distintas ofertas televisivas. En: UCM (ed.), El Debate de la Comunicación. Madrid: Fundación General de la UCM, 1997, pp. 386 - 296.

Los rasgos característicos más importantes que han delineado al sector audiovisual europeo son: el conflicto entre la perspectiva de servicio público en el sector de las telecomunicaciones y el existente en la actualidad, de máxima liberalización y fuerte orientación comercial, lo cual se traducirá, en la mayor parte de los países europeos, hasta el presente, en un fuerte lastre cuando se trata de visualizar la forma de implantación de la televisión digital; un heterogéneo campo de regulaciones en el escenario audiovisual europeo, lo cual ayuda a entender el marco de desigualdad que hay entre los diferentes soportes tecnológicos que hoy contamos para la instauración de la digitalización de las telecomunicaciones en esta región; un escenario europeo en el que las barreras idiomáticas y culturales condicionan la producción de contenidos comunes; las posiciones dominantes o control de grupos empresariales nacionales e internacionales de contenidos con un elevado valor de cambio 
entre los ciudadanos; y un parque de receptores, muchos de ellos, carentes de acceso de recibir la señal digital a menos que se cuenten con descodificadores que permitan tal fin; por citar algunos aspectos que han marcado su evolución.

La televisión digital, al insertarse bajo estas condiciones, es heredera de la historia audiovisual europea. El momento en que transitamos, está determinado por el conflicto y diversidad de desarrollo dispar entre los diferentes procesos y soportes llevados a cabo en el seno de este sistema. Salvo en el caso de la televisión digital satélite, en el que se han generado beneficios ya consolidados, a lo largo de todo su proceso de inclusión en el contexto europeo, el cable y la televisión digital terrestre (TDT) se han caracterizado por sus constantes vacíos y dudas, ante los vaivenes de estrategias que han girado del sistema de transmisión público al privado, así como de la fuerte ruptura del modelo de televisión pública instaurado en el patrón de acceso televisivo entre los ciudadanos europeos, entre otros factores; los cuales trajeron consigo un escenario inicial de números rojos que, progresivamente, ha venido estabilizándose hasta comenzar a reducir las brechas en la zona europea entre soportes, llegándose para finales del 2007, en el caso de la TDT, a romperse la barrera del $50 \%$ de penetración de hogares en contacto directo con esta nueva base tecnológica ${ }^{3}$.

La evolución de la digitalización del sector televisivo europeo, va a estar orientada de acuerdo con la inversión de investigación y desarrollo, pautada en torno a los planes de desarrollo de la sociedad de la información y conocimiento y tecnología de información y conocimiento en cada uno de los países, en especial, en el campo telemático.

El creciente desarrollo del sector de las TIC's en cada uno de los países de vanguardia de la Unión Europea (Reino Unido, Irlanda, Suecia, Noruega y Finlandia), dan cuenta de la visión que tuvieron estos desde el comienzo de este proceso, ante las oportunidades brindadas por sus condiciones culturales, sociales, económicas y geográficas, entre otras, las cuales favorecieron un escenario de promoción más favorable, en comparación al resto de países miembros que se encuentran con un mayor nivel de rezago.

En la mayoría de los casos, muchas de estas previsiones sirven para ubicarnos y ver el perfil de desarrollo que cada soporte ha ido adquiriendo, hasta ahora, en el contexto europeo, las claras diferencias entre sí, así como el re-posicionamiento de cada uno en el escenario regional, ante la inclusión de nuevos actores comerciales dispuestos a invertir y de una normativa con base más sólida a la existente al inicio de este proceso.

Si analizamos la dinámica económica en la televisión digital en Europa, apreciamos que la situación en este aspecto no se ha caracterizado hasta ahora por ser muy brillante que se diga.

La dinámica, por ejemplo, en torno a la TV digital por satélite en la región muestra cómo ha sido requerida la fusión de los principales operadores europeos, para enfrentar el elevado nivel de inversión requeridas para su puesta en marcha ${ }^{4}$. Por ello,

\footnotetext{
3 Véase http://www.elmundo.es/elmundo/2007/10/03/comunicacion/1191400678.html
} 
el escenario satelital está regido por procesos de fusión concluidos o en vías de realizarse, según el país de estudio, así como la constante presencia de un inestable equilibrio económico, aún por alcanzar ${ }^{5}$.

Con respecto al cable, el proceso descrito se agudiza más, ante las desventajas implícitas en este soporte, producto de las elevadas inversiones requeridas para la construcción de la estructura técnica, su condición eminentemente local para su desarrollo comercial, entre otros factores. Estas limitaciones han traído consigo que, pese a la honda historia de este soporte a nivel mundial y de sus ventajas para el desarrollo de servicios interactivos, en Europa aún no se haya generalizado y/o asentado de igual forma que el satélite. Salvo algunos países puntuales de la Unión Europea, donde su condición geográfica y apuesta más seria en el cable, desde la década de 1950 y 1960, han permitido un mayor impacto ${ }^{6}$. El desarrollo de este soporte va a estar enfocado más hacia el establecimiento de monopolios en las zonas de adscripción con muchos problemas de recepción de otro tipo de señal.

La TDT, pese a su condición de inicio dubitativo, ha empezado a convertirse en la principal apuesta al hablar de televisión digital a nivel europeo, ante la visión que se tiene alrededor de ella y el paso definitivo hacia la digitalización total de la región; dejando atrás lo analógico del escenario audiovisual. Detrás del interés por el desarrollo de la TDT en Europa existe además la posibilidad de usar, más eficientemente, la frecuencia radioeléctrica en la región. Recurso, actualmente, colapsado ante su carácter finito y, por ende, limitado.

Con el afianzamiento de este sistema, se hará un uso más efectivo del bien radioeléctrico de cada país y región del mundo y, con ello, se ampliarán las posibilidades de desarrollo de nuevas actividades económicas en el área de las telecomunicaciones, así como de nuevas vías de acceso potencial de información y conocimiento, a través del desarrollo de nuevos servicios integrados, producto de la convergencia e interacción tecnológica que comienza a visualizarse cada vez mejor entre lo tradicional, la dieta audiovisual del modelo tradicional y la capacidad de acceso a través del televisor de la Internet, entre otros soportes, las cuales se encuentran limitadas por su imposibilidad de apropiación de otras frecuencias para ello. Al menos, hasta que no se termine de concretar el paso definitivo de lo analógico a lo digital del sector audiovisual en Europa y el resto del mundo. A partir de este proceso de transición, se irá asentando un horizonte donde lo multimedia y la hipertextualidad terminarán cobrando más realidad. Esto permitirá que lo audiovisual comience a contar con la oportunidad de ser accedido a través de soportes ajenos a la tradicional televisión en casa?.

\footnotetext{
${ }^{4}$ Ejemplo de ello lo tenemos en España, Francia, Italia, Alemania, por citar algunos países.

5 Quizás Reino Unido sea uno de los pocos casos, en torno a esta modalidad de televisión digital, en el cual se ha percibido resultados positivos desde su comienzo.

6 Tal es el caso de Alemania, donde más del $80 \%$ de la población tiene acceso por cable.

7 Un claro ejemplo de lo aquí expuesto lo tenemos en el desarrollo y paulatino proceso de inserción de los móviles de tercera generación [3G], los cuales desde mediados de 2006 han comenzado a ser ofertados por las principales compañías de telecomunicaciones europeas; generando esto el inicio de un nuevo proceso de aproximación de lo audiovisual por medio de este soporte.
} 
Con el desarrollo de este nuevo sistema en la región, no sólo la Comisión Europea y las administraciones públicas han asumido el reto de avanzar en torno a la universalización de acceso a la información por un mayor número de personas, por medio de lo digital, sino que también, el interés y las expectativas de los diferentes actores económicos del sector telemático, sobre todo los provenientes del área de la telefonía móvil, han ejercido presión a favor de una transición que garantice concretar, más rápido, las potenciales oportunidades de ampliación de sus beneficios económicos, a través de la nueva gama de servicios que pueden ser ofertados, transversalmente, desde los diferentes soportes que actualmente existen en el mercado a disposición de los ciudadanos en capacidad de pago y empleo de estas oportunidades.

Pese a este escenario de compromiso asumido desde los diferentes actores involucrados en el proceso de digitalización en Europa, aún existe un elevado número de incertidumbres, a saber:

1. El modelo económico requerido para el sostenimiento de la televisión digital en el conjunto de la región, sobre todo en lo concerniente a la TDT $^{8}$.

2. El conjunto de obstáculos que hay que resolver en el campo de la oferta de servicios integrados desde los diferentes soportes que comienzan a surgir ante el avance de lo digital del sector audiovisual, ante la fuerte inversión necesaria para la actualización de las redes de difusión y la transformación del canal analógico que antes era empleado para la transmisión de contenidos audiovisuales, a cinco nuevos canales digitales, con capacidad técnica para la interacción de nuevos servicios y contenidos, provenientes de soportes hasta ahora ajenos (Internet, telefonía, entre otros).

3. La fuerte inversión requerida para la producción de contenidos que atraigan y marquen una diferencia cualitativa suficiente para que una mayor cantidad de personas estén dispuestas a llevar a cabo la cuota de inversión de adquisición de los nuevos receptores.

4. La generación de estrategias promocionales que den a conocer las ventajas de este nuevo soporte y, así, se logre marcar una diferencia que le permita a la TDT ganar un espacio en el complejo mercado competitivo existente en la actualidad, con los operadores satelitales y por cable ya establecidos.

\footnotetext{
8 Tal es el caso del fracaso presentado en España cuando se lanza QuieroTV, el cual tuvo que cerrar por su incapacidad de hacer frente al elevado nivel de deuda adquirido desde su lanzamiento en 2002 y el modelo comercial de pago asumido en él, que devino en su entrada al mercado donde la elevada cuota de control de los operadores de TV de pago por satélite (Vía Digital y Canal Satélite Digital), así como de los diferentes operadores por cable existentes, trajo consigo no solo asumir la dificultad propia de todo negocio en sus primeros momentos, sino un elevado nivel de competencia por un número reducido de abonados potenciales. Otro ejemplo es el caso del R. Unido, donde ITV Digital tuvo que regalar los decodificadores y cambiar el modelo comercial de pago a un modelo de Freeview, sostenido con el pago de impuesto o canon que recibe la BBC y, también, formar una alianza con uno de los principales operadores satelitales en dicho país BSkyB TV para evitar su cierre y obtener un mayor nivel de abonados.
} 
Existen muchas incertidumbres aún en torno a la digitalización en el ámbito europeo. El requerido proceso de inversión económica en el campo promocional, de contenidos, entre otros; ha hecho que la opción de la vía administrativa, desde la Unión Europea y mayoría de países miembros, haya sido tomada como mecanismo de impulso inicial para impulsar el paso de lo analógico a lo digital en el campo audiovisual. Esta estrategia ha generado un escenario de imprevisión y constantes cambios normativos y técnicos, cuando la mayoría de los países aún no poseen las condiciones propicias para ello, pese a los fuertes avances logrados en los últimos años.

En la mayoría de los países de la UE, el período concerniente al apagón analógico oscila entre 2008-2012, siguiendo lo establecido por la Comisión Europea para este asunto. Este tiempo optado luce más el deber ser que el ser real, ya que el avance y posibilidad de inversión generado por los usuarios para el cambio de receptores, pese a contar actualmente con decodificadores que permiten el acceso a la señal de TDT presenta rezagos, ya que aún no se han creado las condiciones idóneas para el fortalecimiento de los incentivos necesarios para que las familias vean en estos nuevos soportes una oportunidad de acceso a un mayor horizonte de ventajas.

El proceso de adquisición tecnológica requerido para el paso definitivo de lo digital en el contexto audiovisual, en general, ha sido lento y dependiente del coste económico requerido para ello en cada país y región.

El desarrollo de los servicios generados ante el proceso de digitalización del sector audiovisual, así como de factores relacionados para su concreción, promoción o difusión desde los estados y actores económicos, capacidad de generación de un marco económico saludable de competencia entre los actores empresariales telemático, el proceso de alfabetización digital del ciudadano europeo para el uso de los nuevos recursos que comienzan a abrirse paso, y la generación de un escenario de apoyo institucional, nacional y regional, para el acceso a dichos recursos, por parte de un mayor porcentaje de la población, entre otros factores; contribuirá a solventar los constantes pasos en falso que se han venido generando, ante la toma apresurada de decisiones políticas cuando no se daban las condiciones reales para el paso definitivo de lo digital ${ }^{9}$.

Hasta que lo antes expuesto no se termine de concretar, el proceso de cambio tecnológico para el acceso a la televisión digital en Europa, terminará por trascender, en muchos países de la UE los períodos ya pautados, llegando incluso a rozar o superar la fecha establecida por este organismo en 2012. Es por ello que es necesario que se amplíe el rol de las administraciones al respecto, es decir, ampliar las vías para la instauración de planes gubernamentales reales, en los que se subvencione, total o parcialmente, la adquisición de los equipos requeridos, por parte de los ciudadanos $^{10}$, así como se fomenten de mayores canales de incentivos comerciales a

\footnotetext{
9 Tal es el caso de Italia, país que tenía pautado el apagón analógico para 2008 y que tuvo que postergar para 2012 este proceso por considerar poco realista el plazo ya establecido (Satcesc, http://www.satcesc.com/noticia/octubre2007/015.htm).

10 Italia, el gobierno ha establecido ayudas económicas para la adquisición de comprar estos aparatos para un limitado grupo de personas (1.000.000 de familias), destinando un porcentaje de lo generado por la privatización de la televisión pública. Otro ejemplo es el Reino Unido, donde los operadores asumen la inversión en este aspecto, financiando la compra de estos decodificadores.
} 
los actores económicos llamados a apostar por este nuevo escenario y en la instrucción digital de las personas que forman parte de las diferentes sociedades en las que se enmarca Europa, sobre todo entre aquellos ajenos a lo que se conoce como generación bit (Sartori, 2002).

En Europa, la IPTV desde 2004 ha comenzado un lento proceso de desarrollo, en el que, lo más notorio, es el actual estado de insipiencia a la hora de determinar el verdadero impacto que tendrá a nivel europeo (Muñiz). Ello, a pesar de no ocultar los favorables rasgos técnicos con que cuenta esta tecnológica de transmisión audiovisual, de cara a ganarse un puesto en el espacio audiovisual europeo. En 2006 este soporte aún se encuentra en la fase inicial en Europa, siendo Suecia uno de los principales países europeos con un importante terreno avanzado en torno a la IPTV, al tener para esa fecha ya cuatro canales bajo esta modalidad de transmisión ${ }^{11}$ (Cullen).

Este soporte cuenta con grandes saltos cualitativos y cuantitativos a la hora de dibujar el mapa de penetración de este soporte en el contexto europeo, pues son los principales países de la Unión Europea de los 25 países quienes lideran su inclusión en la región (Italia, Reino Unido, Francia, España, y Alemania).

A pesar de los avances que se han experimentado en 2006 y comienzo de 2007, la estructura europea de la IPTV se encuentra, en muchos casos, carente de una base reguladoras 0 , en los mejores escenarios, desarrollado bajo las amplias leyes de telecomunicaciones existentes en cada país, las cuales favorecen el progreso de este sector en esta fase inicial. De todas formas, resulta pronto establecer las dimensiones reales que terminará adquiriendo la televisión por Internet en esta región, sobre todo, teniendo en cuenta que su instauración requiere del proceso de cambio de la infraestructura tecnológica requerida para tal fin y de la ampliación del acceso y uso de Internet en los hogares europeos, así como de los patrones de consumo tecnológico de los ciudadanos que residen en esta región.

Son hoy muchas las brechas existentes en este tema entre los países de la UE, y en muchos casos para 2006 aún no existían ofertas audiovisuales enmarcadas en este soporte (ejemplo tenemos en Luxemburgo, Grecia y Portugal).

Lo que hoy tenemos es un nuevo sector que requiere ser regulado bajo una normativa en la que se amparen los diferentes campos de acciones y oportunidades a desarrollarse en él, así como también es necesario que se den unas reglas claras para que los diferentes operadores que comienzan a surgir desde el sector tradicional de televisión en cada país, y en el campo de la telefonía, muestren un mayor interés de ampliar su oferta comercial con el ofrecimiento de TV y servicios enriquecidos bajo este soporte.

\section{LO DIGITAL EN EUROPA. ESPACIO DE REFLEXIÓN Y DEBATE}

El desarrollo de lo digital en esta región está en un momento de elevada expectativa, ante las diversas modalidades de configuración y la forma como se están llevando a cabo los procesos de ajustes entre los canales privados en abierto, los de pago y los canales públicos, ante la mirada y búsqueda de un marco favorable para

\footnotetext{
11 TeliaSonera, Canal Digital, FastTVnet, Bredbandsbolaget.
} 
el nuevo contexto y abanico de oportunidades que ella pudiese brindar, no sólo a las empresas enmarcadas en el sector telemático, sino también al ciudadano.

El principal elemento diferenciador es que en el caso de Europa, la complejidad es mayor, al estar constituida la región por muchos países con realidades sociales y culturales distintas. Ello trae consigo un crecimiento del sector televisivo digital de forma variante y con niveles de mayor o menor expectativas e incertidumbre, de acuerdo con la condición de cada uno y con las obligaciones impuestas en el contexto europeo, las cuales ejercen presión reguladora del desarrollo de este mercado, con el fin de homogeneizar el sector audiovisual en la región ${ }^{12}$, así como en la forma de incluir en este proceso de transición al ciudadano.

El desarrollo actual de la televisión digital es diverso y, en general, salvo los casos del satélite y cable que poseen más tiempo de instauración y, por ende, de análisis y de asentamiento en el contexto regional y mundial, la mayoría de incertidumbres que se tienen en el presente están siendo producidas por las diferentes estrategias de asentamiento de la TDT y del IPTV.

La realidad fragmentada de la Unión Europea imposibilita, desde nuestro punto de vista, unificar criterios comunes y técnicos tan fácil y rápido como el avance que requiere el campo telemático regional. La realidad social y cultural de cada país; el nivel de coherencia de las diferentes administraciones a la hora de establecer los marcos normativos, bajo la influencia del interés político y económico en ese momento; las posiciones de ventajas competitivas de operadores nacionales y su consiguiente impacto en la inclusión de nuevos actores, al poseer un control en los principales contenidos estratégicos del sector y del mayor número de abonados en ellos; así como la capacidad de estos actores comerciales de realizar la fuerte inversión económica a lo largo del proceso de asentamiento digital; son algunos de los principales puntos que marcan el particular ritmo que la televisión digital tiene en Europa y, sobre todo, permite entender el fuerte grado de desigualdad en ella, alrededor de este proceso.

Con el avance tecnológico se aprecia cómo se profundiza más los rasgos antes expuestos en torno al escenario audiovisual y social de Europa. El actual reto que surge con el paso de lo analógico a lo digital, ante esta realidad, hace que no se potencien aún más las fortalezas y cualidades existentes en el contexto cultural, social y empresarial en esta zona del planeta.

La creciente disposición de cada Estado en asumir un rol pasivo en este complejo puzzle audiovisual ha generado que la sensación de vacío sea una constante y que se asuma la transición completa de lo analógico a lo digital como algo definitivo, sin tener presente las estrategias que permitirán ello de forma realista, en muchos casos, cumplido el plazo propuesto o asumido por cada gobierno europeo para evitar así

\footnotetext{
12 Ejemplo de algunas de las medidas impuestas en el ámbito de la UE: Los principios y directrices de la política comunitaria en el sector audiovisual en la era digital y la participación de la Comisión Europea en el Observatorio Europeo del Sector Audiovisual o la directiva de Televisión Sin Frontera. En ambos casos, el objetivo ha estado orientado a la definición de las prioritarias en la UE para comienzo del siglo XXI, la elaboración de objetivos y principios de la política comunitaria en el sector audiovisual a medio plazo, el aprovechamiento de las diferentes ventajas que tanto se promueven en torno a este proceso y el seguimiento en torno a la evolución del mismo.
} 
quedarse detrás en un proceso que está llamado a acentuar los importantes cambios que está ejerciendo, no sólo en lo referente al ámbito empresarial, sino también en torno a la reconfiguración del mapa comunicacional y cognitivo de los individuos que dan cuenta de la sociedad (Said, 2006).

\section{REFERENCIAS BIBLIOGRÁFICAS}

ÁLVAREZ, J. Las distintas ofertas televisivas. En: UCM (ed.), El Debate de la Comunicación. Madrid: Fundación General de la UCM, 1997, pp. 386-396.

ARNANZ, C. Negocios de televisión. Transformaciones del valor en el modelo digital. Barcelona: Editorial Gedisa, 2002.

ASOCIACIÓN ESPAÑOLA DE EMPRESAS DE TECNOLOGÍA DE LA INFORMACIÓN. Métrica de la sociedad de la información. [documento electrónico] 17 de julio de 2006. [Consulta: 20 de noviembre de 2006]. <http://www.aetic.es/VerDocumentos.aspx?id=58\&idContenidos $=103>$

BECK, U. ¿Qué es la globalización? 1ª ed. Barcelona: Editorial Paidós, 1998.

BRÜNNER, J. J. Globalización, cultura y postmodernidad. $1^{\mathrm{a}}$ ed. Santiago, Chile: Editorial Fondo de Cultura Económica, 1998.

COMISIÓN DEL MERCADO DE LAS TELECOMUNICACIONES. Informe anual 2006. [informe electrónico] 5 de febrero de 2007. [Consulta: 27 de marzo de 2008]. Disponible en http://www.cmt.es/cmt ptl ext/SelectOption.do?nav=publi anuales.

CASTELLS, M. La Era de la Información: Economía, Sociedad y Cultura: I La Sociedad en Red. Vol 1. 2a ed. Madrid: Editorial Alianza, 2005.

CULLEN INTERNATIONAL. Cross-country analysis - Western Europe. IPTV commercial offers [documento electrónico]. 1 de Julio de 2006. [Consulta: 25 de marzo de 2008]. Disponible en <http://www.itu.int/osg/spu/ni/voice/documents/Background/IPTV_Cullen_International.pdf $>$

COMISIÓN EUROPEA. Europe's Information Society [en línea]. [Consulta: 27 de marzo de 2008]. Disponible en <http://ec.europa.eu/information_society/index_en.htm>

DOBARRO, S. La Televisión Digital en Europa. $1^{\text {a }}$ edición. Santiago de Compostela: Editorial 9, 1998.

DVB. [portal electrónico]: [Consulta: 28 de marzo de 2008]. Disponible en $\leq$ http://dvb.org/ >

EL MUNDO. La TDT avanza con paso firme en Europa. [en línea]. 3 de octubre de 2007. [Consulta: 27 de marzo de 2008]. Disponible en <http://www.elmundo.es/elmundo/2007/10/03/comunicacion/1191400678.html> 
FUNDACIÓN TELEFÓNICA. Digiworld. América Latina 2007 [digital]. Madrid: 2007. 26 marzo 2008, [Consulta: 26 de marzo de 2008]. Disponible en <http://www.fundacion.telefonica.com/forum/Digiworld/\#>

- (2005). Las Telecomunicaciones y la Movilidad en la Sociedad de la Información. Madrid.

FOGEL, J. F. La calidad periodística como factor de mercado: la búsqueda de la credibilidad e independencia de las empresas. En: CAF (ed.), Ética, calidad y empresa periodística en América Latina. Caracas, Venezuela: CAF y Fundación Nuevo periodismo Iberoamericano, 2004, pp. 35-49.

GARCÍA CANCLINI, N. Culturas Híbridas. Buenos Aires, Argentina: Editorial Paidós, 2001.

GIDDENS, A. Un mundo desbocado. $1^{\text {a }}$ ed. Madrid: Editorial Taurus, 2002.

INSTITUTO NACIONAL DE ESTADÍSTICA. Acceso a Internet de las viviendas por valor absoluto/porcentaje, características del hogar y forma de conexión a internet. [en línea]. 12 de marzo de 2006. [Consulta: 12 de marzo de 2006]. Disponible en $\leq$ http://www.ine.es/inebase/cgi/axi >.

INTEL. Moore's Law. [en línea]. 26 marzo 2008, [Consulta: 26 de marzo de 2008]. Disponible en <http://www.intel.com/technology/mooreslaw/index.htm>

INTERNATIONAL TELECOMMUNICATION UNION. Measuring the Information Society 2007. $1^{\text {a }}$ ed. Estados Unidos: Autor, 2007.

- (2007a). Trends in telecommunication reform 2007. Estados Unidos: Autor.

- (2005). The digital guide to the digital opportunities. Québec, Canadá: Autor.

OLALQUIAGA, C. Megalópolis. $1^{\text {a }}$ ed. Caracas. Venezuela: Monte Ávila Editores, 1991.

MUÑIZ, I. Televisión IP: Una Experiencia Totalmente Personalizada [en línea]: Centro de Investigación e innovación en Telecomunicaciones (CINIT). 15 de julio de 2005. [consulta: 22 de octubre de 2006] Disponible en $\leq$ http://www.cinit.org.mx/articulo.php?idArticulo=34>

NEGROPONTE, N. El mundo digital. $1^{\text {a }}$ ed. Barcelona: Ediciones B, 2000.

PILATI, A. El mercado televisivo frente a la revolución digital. En: UCM (ed.), El Debate de la Comunicación. Madrid: Fundación General de la UCM, 1997, pp. 397-403.

RAMONET, I. La golosina virtual. Madrid, España: Editorial Debate, 2000.

REVISTA TELEDIGITAL. Anuario 2006. $1^{\text {a }}$ ed. Núm 9. Año 9. Madrid: Tele satélite publicaciones España S.L. 
ROJO, P. La televisión digital en Europa: el difícil equilibrio entre la liberalización del sector y el respeto a la diversidad cultural y lingüística de los Estados miembros. En UCM (ed.). La Radio y la Televisión en la Europa Digital. Madrid: Facultad de Ciencias de la Información, 2005, pp. 65-78.

ROBERTSON, R. Glocalización: tiempo-espacio y homogeneidad heterogeneidad. Cholonautas [en línea] 29 de octubre de 2007. [consulta: 29 de octubre de 2007]. Disponible en <http://www.cholonautas.edu.pe/modulo/upload/R\%20Robertson.pdf>

SACRISTÁN, C.H. Interculturalidad, transculturalidad y valores de la acción comunicativa. En GRUPO CRIT (Eds.). Claves para las comunicaciones intercultural. Barcelona: Publicacions de la Universitat Jaume I, 2003, pp. 17-35.

SAID, E. M. La Convergencia Tecnológica en los Servicios Ofertados en la TV de Pago y su Impacto en el Mapa Comunicacional. [tesis doctoral] $1^{\text {a }}$ ed. Madrid: Universidad Complutense de Madrid. 2006. ISBN: 978-84-669-2986-8.

- (2005). "La plutarquía mediática. Nuevas estrategias de alianzas económicas e impactos en la Sociedad de la Información". Revista Contratexto Digital [revista electrónica]. 15 junio de 2005, $n^{\circ} 3$. [consulta: 15 de junio de 2005]. Disponible en <http://www.ulima.edu.pe/Revistas/contratexto/art8.htm>

SARTORI, G. Homo videns. La Sociedad teledirigida. $4^{\mathrm{a}}$ ed. Madrid: Editorial Taurus, 2003.

SANTOYO, A.; MARTÍNEZ, E. La brecha digital. Mitos y realidades. $1^{\text {a }}$ ed. México D.F., México: Universidad Autónoma de Baja California, 2003.

SATCESC. Las emisiones de televisión en este país europeo se aplazan cuatro años sobre la fecha prevista, en diciembre de 2008, hasta el año 2012, dos años después del plazo previsto en España. [en línea]. 26 marzo 2008, [Consulta: 26 de marzo de 2008]. Disponible en <http://www.satcesc.com/noticia/octubre2007/015.htm>.

VILCHES, L. La migración digital. 1ª . ed. Barcelona: Editorial Gedisa, 2001.

"La TV española de pago no avanza". Revista Teledigital. Madrid: Tele satélite publicaciones España S.L. n 126, p. 24.

\section{Breve semblanza del autor}

Elias Said es Licenciado en Sociología por la Universidad Central de Venezuela (2001), y doctor en tecnología, estructura y tratamiento de la información por la Universidad Complutense de Madrid (2006). En la actualidad es profesor de la Universidad del Norte de Barranquilla, donde ejerce labores docentes e investigadoras en el programa de pregrado y postgrado en el área de la comunicación.

(Recibido el 16-04-07, aceptado el 21-03-08) 